\title{
PAPILLOMA OF THE FOURTH VENTRICLE *
}

\author{
REPORT OF A CASE \\ ERNEST SACHS, M.D. \\ ST. LOUIS
}

A butcher, 50 years old, complained of headache, dizziness and falling on walking. The past history was unimportant. Two and a half years ago he began to have headaches and pain in the lower part of the abdomen. One year ago he had an attack of influenza. During this period his headaches were less severe. During the two months before admission he had intense headaches accompanied by vomiting without nausea and was unable to walk without help. He fell to either side, but most frequently forward. During the past two weeks he had several attacks of unconsciousness lasting from two to ten minutes, unaccompanied by convulsions or twitchings.

The positive findings in the physical examination were: questionable lateral nystagmus to both sides, at times a lateral nystagmus which was quite definite with a slow component to the right, the head held to the right in a cerebellar attitude, hypesthesia of both corneas, more on the right, and normal eyegrounds and visual fields. The visual acuity was: left $20 / 60$, right 20/48. The pupils were equal and reacted to light and accommodation.

There was some cerebellar ataxia, the patient walking with a broad base. When he attempted to stand on either foot he reeled to the right and backward. There was hypotonia of the right leg. There was no adiadokokinesis in either hand; finger to nose test and all tests to determine finer movements of the fingers were normal. There was a history of regurgitation through the nose and difficulty in swallowing. Roentgen-ray examination was negative. Lumbar puncture showed 13 cells, ++ Pandi and a negative Wassermann test. The only abnormalities that the Bárány tests showed were that when the right ear was douched with hot water the patient did not past point with the left hand, when the horizontal canals were tested; when the vertical canals were tested with the patient's head back the patient always past pointed to the left with both hands. Dr. Lyman's comment on these tests was as follows: "The vestibular tests suggest a lesion in the brain stem in the region of the posterior longitudinal bundles. The cerebellum gives practically normal reactions."

* Read at the Forty-Eighth Annual Meeting of the American Neurological Association, Washington, D. C., May, 1922. 


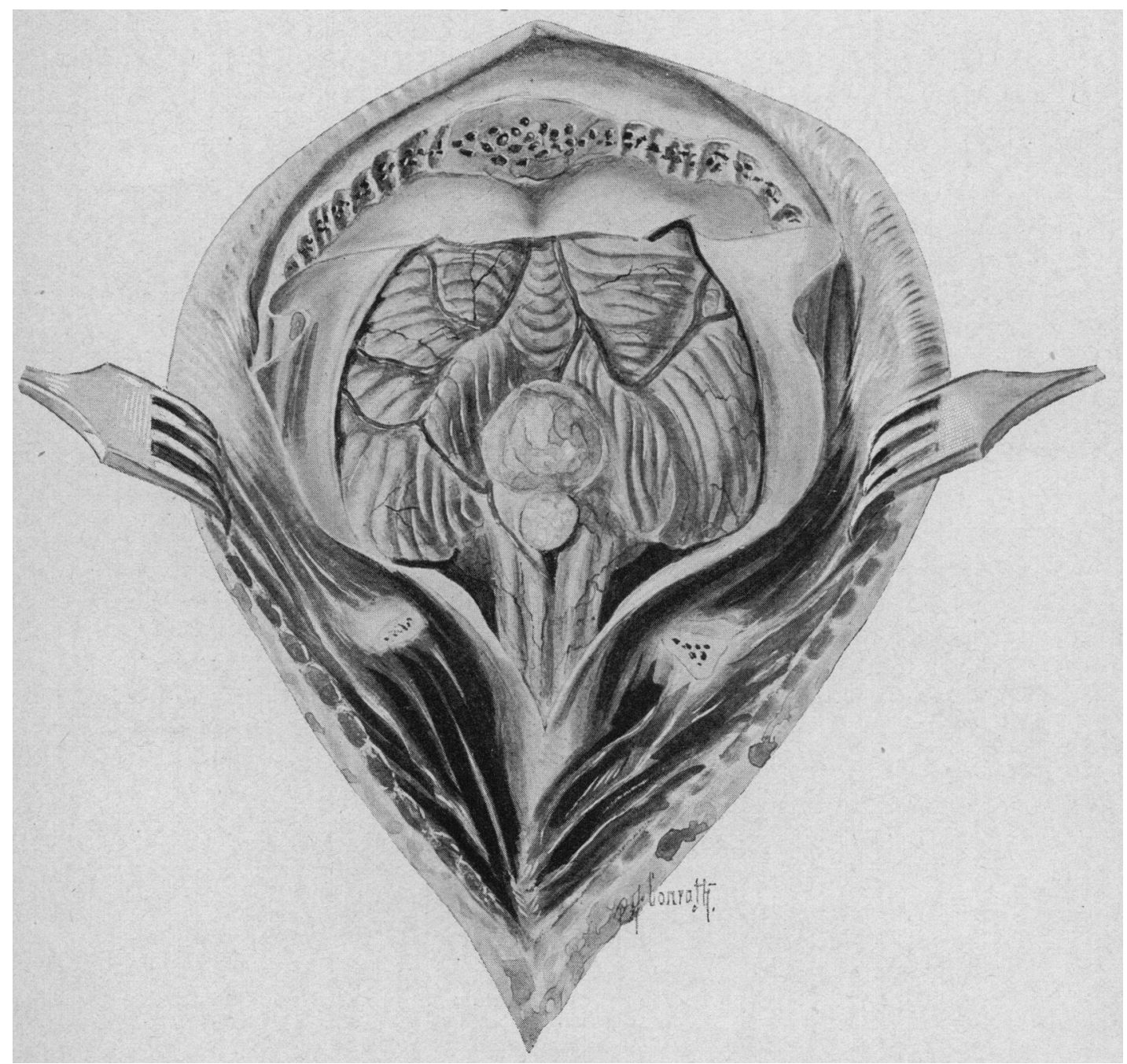

Fig. 1.-Papilloma of the fourth ventricle removed as shown in Figure 2, under local anaesthesia, through a median line incision. The tumor extended up into the aqueduct of Sylvius. Complete recovery with disappearance of all symptoms. 


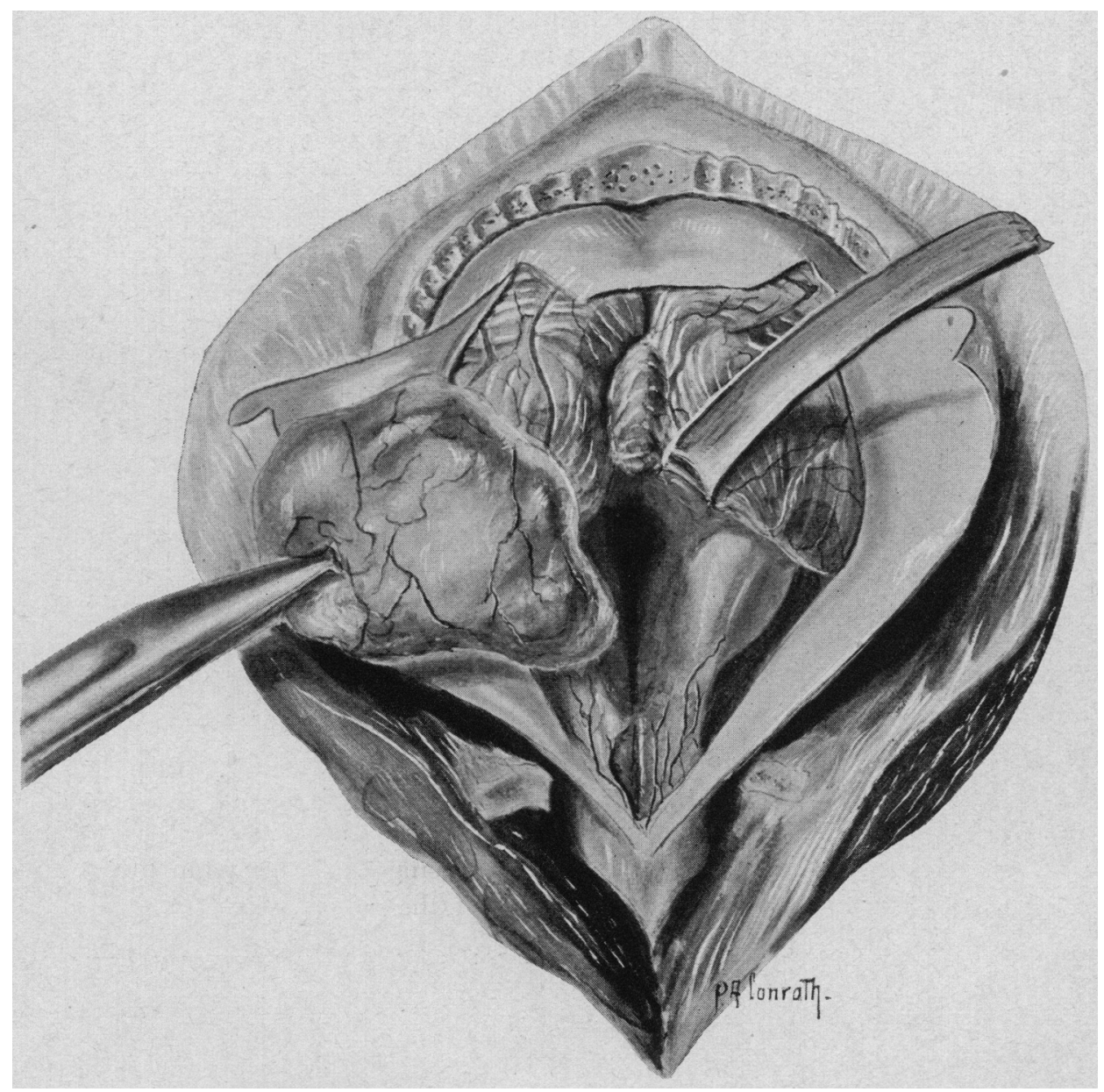

Fig. 2-Removal of papilloma of fourth ventricle. 
The symptoms were so clearly bilateral that it seemed quite probable that the lesion lay in the median line. In view of the occupation of the patient, Dr. Schwab and I both thought of the possibility of a cysticercus infection. The absence of nystagmus suggested that the lesion was superficial and not near the nuclei of the cerebellum. The absence of choked disk suggested that the lesion was growing slowly.

In view of the probable median line location of the process, the usual cross bow cerebellar exposure was not employed; merely a median line incision was made with retraction of the muscles and removal of the occipital bone and the arches of the atlas and axis. This was done under local anesthesia and gave an excellent exposure of the fourth ventricle and the vermis. On opening the dura a white glistening tumor was seen filling the fourth ventricle. This was well encapsulated and was enucleated without much difficulty and without pain to the patient. The tumor extended up into the aqueduct of Sylvius, which was greatly dilated. The only discomfort complained of during the operation was pain in the abdomen. The patient made an uneventful recovery and left the hospital on the eighteenth day after operation. Now, a year after the operation, he is entirely free of symptoms with the exception of slight ataxia when he walks rapidly up stairs.

The three reasons for presenting the case are: 1 . The constant pain in the abdomen, which I believed might be interpreted as evidence that there are afferent fibers in the vagus nucleus. This abdominal pain was present both before operation and when the tumor was lifted from the floor of the fourth ventricle. 2. The tumor had completely obstructed the aqueduct of Sylvius and therefore produced an obstructive hydrocephalus, and yet the patient had normal eyegrounds. 3 . This case demonstrates the possibility of removing tumors of considerable size in the region of the fourth ventricle through a simple median line incision without freeing the muscles from their attachment to the superior curved line of the occipital bone.

\section{DISCUSSION}

Dr. Foster Kennedy, New York: Abdominal pain similar to that associated with this tumor is often found in vasovagal attacks, confirmatory evidence of the involvement of the vagus in seizures of that type. 